\title{
Clinical and magnetic resonance imaging study of extrapyramidal symptoms in multiple system atrophy
}

\author{
Masaaki Konagaya, Yoko Konagaya, Mitsuo Iida
}

\begin{abstract}
Slit-hyperintensity in the outer margin of the putamen on T2 weighted MRI was found in 17 out of 28 patients with clinically diagnosed multiple system atrophy. Thirteen of these 17 patients showed extrapyramidal signs. Five patients had only unilateral slit-hyperintensity; four of them had contralateral rigidity; and one had bradykinesia. Despite mild rigidity, one case showed no slit-hyperintensity. One of the 14 cases with parkinsonism showed no hyperintensity, and four of the 14 cases without parkinsonism showed hyperintensity. On the other hand, slit-hyperintensity was not seen in any of 25 patients with clinically diagnosed Parkinson's disease. Putaminal slit-hyperintensity is a useful MRI feature in the differential diagnosis between Parkinson's disease and multiple system atrophy predominantly affecting the extrapyramidal system.
\end{abstract}

(F Neurol Neurosurg Psychiatry 1994;57:1528-1531)

Multiple system atrophy ${ }^{1}$ may present with cerebellar, extrapyramidal, or autonomic nervous symptoms and signs. Those patients who primarily manifest extrapyramidal symptoms and signs are often labelled as having striatonigral degeneration, and this presentation is often difficult to differentiate clinically from Parkinson's disease. The main pathological findings of striatonigral degeneration are neuronal loss, demyelination, and gliosis, principally in the putamen and substantia nigra. ${ }^{23}$ Most patients with multiple system atrophy whose initial or predominant symptoms and signs are usually cerebellar, also develop extrapyramidal features in the course of the illness, and other patients who show no extrapyramidal symptoms in life often have pathological evidence of subclinical striatonigral degeneration. ${ }^{4}$

In patients with striatonigral degeneration, signal intensity in the putamen decreases on T2 weighted MRI, ${ }^{5-7}$ giving rise to slit-hyperintensity in its lateral margin. Although this fact has been documented in previous reports, ${ }^{89}$ there have been no specific studies on clinical and MRI correlations for this abnormal finding. We have therefore evalu- ated T2 weighted images of patients with clinically diagnosed multiple system atrophy and patients with Parkinson's disease to clarify the relation between the slit-hyperintensity and clinical features.

\section{Patients and methods}

Twenty eight patients with clinically diagnosed multiple system atrophy (13 men and 15 women, mean age 58.3 (SD 5.4) years were studied. For a clinical diagnosis, patients with multiple system atrophy had to have symptoms or signs of disease in two or all of the cerebellar, extrapyramidal, and autonomic systems. If cerebellar signs were not definitely present clinically, atrophy of the pontocerebellar system on MRI was taken as evidence of cerebellar pathology. In fact, all 28 patients had either cerebellar signs or MRI evidence of pontocerebellar atrophy, or both. All patients had sporadic disease and their clinical course was progressive.

T2 weighted 0.5 or $1.5 \mathrm{~T} \mathrm{MRI}(0.5 \mathrm{~T} 15$ cases; $1.5 \mathrm{~T} 13$ cases) were used to examine abnormal slit-hyperintensity in the outer margin of the putamen. This sign was classified as absent $(-)$, mild $(t)$, moderate $(++)$, severe $(+++)$, or very severe $(++++)$, and then compared with clinical symptoms and signs. Atrophy of the pontocerebellar system on T1 weighted MRI was also rated in a similar manner. Proton density MRI was examined in 22 cases.

Brain MRI was also examined in 25 patients with Parkinson's disease (10 men and 15 women, mean age 64.5 (SD 8.6) years; $0.5 \mathrm{~T} 11$ cases; $1.5 \mathrm{~T} 14$ cases) clinically diagnosed on the basis of clinical features and response to levodopa. Patients with parkinsonism due to other causes were excluded.

\section{Results}

Table 1 shows clinical features, course of illness, and MRI signs in 28 patients with multiple system atrophy. Initial symptoms were ataxia in 13 cases, bradykinesia in 10, and orthostatic hypotension in five. At the time of examination, 14 cases showed extrapyramidal signs such as bradykinesia or rigidity, and in two of them rigidity and akinesia was so severe that the presence or absence of ataxia 


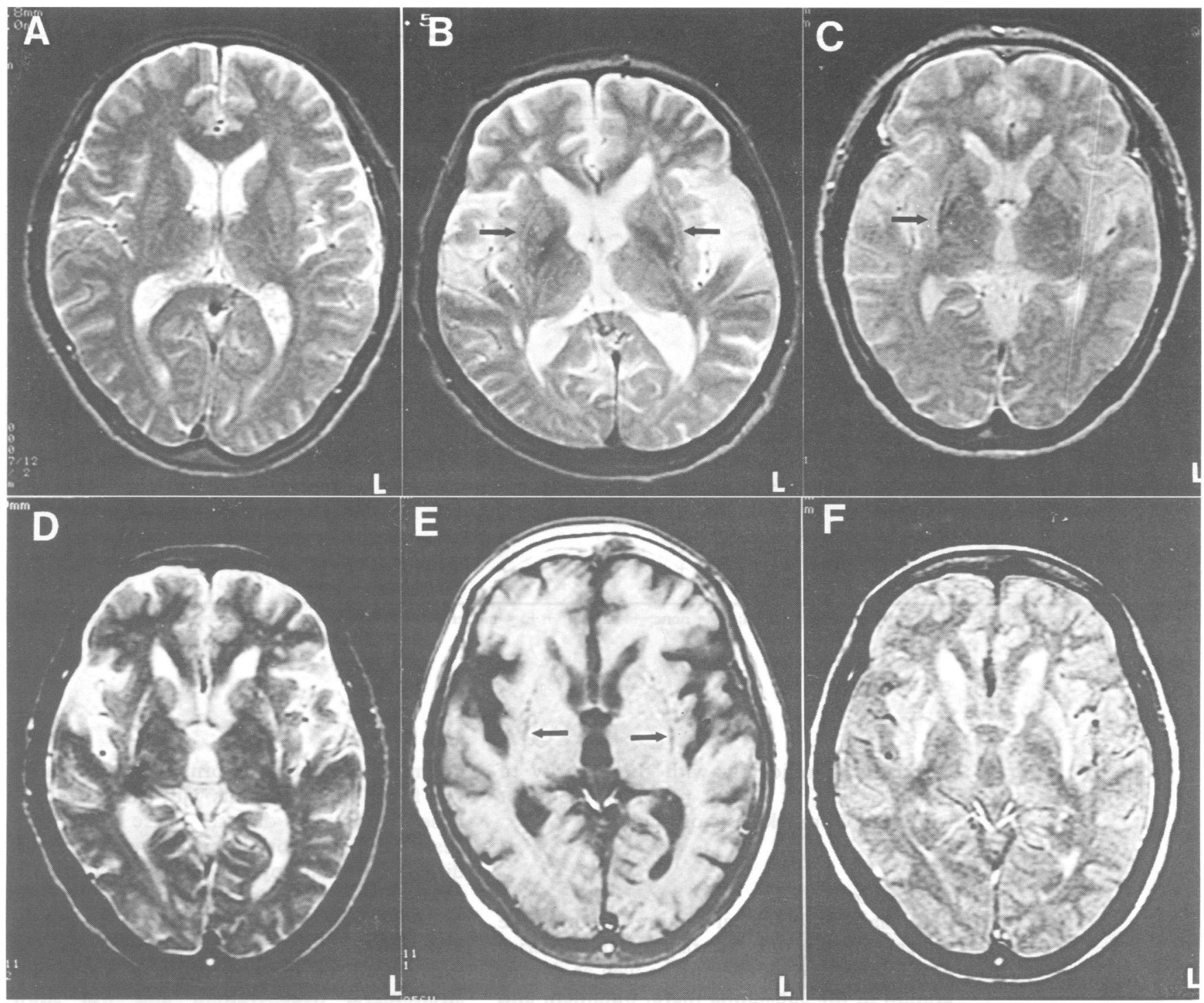

(A) T2 weighted MRI of case $8(0.5 T, T R 2800, T E 100)$. Clinically this patient showed no extrapyramidal signs and no slit-hyperintensity on MRI. (B) T2 weighted MRI of case $24(1.5 T$, TR 2000, TE 80). Clinically, this patient showed moderate limb rigidity on the left side and mild rigidity on the right; MRI showed slit-hyperintensity in the outer margin of the putamen, moderate on the right side and mild on the left (arrows). (C) T2 weighted MRI of case $23(0.5 T, T R 2800, T E$ 100). Clinically, this patient showed rigidity in left limbs; MRI showed contralateral slit-hyperintensity (arrow). (D) T2 weighted MRI of case 27 ( $0.5 T$, TR 2800, TE 100), with severe rigidity, akinesia, and dysautonomia; MRI showed bilateral linear hyperintensity at the lateral margin of the putamen. (E) T1 weighted MRI of case 27 (TR 400, TE17). Hypointense lines were present at the lateral margin of the putamen (arrows). There were similar findings in cases 17, 19,20,23,24, 26, 27, and 28. (F) Proton density MRI of case 27 (TR 2800 , $T E$ 35). Increased signal intensity was present bilaterally in the putamen and caudate nucleus. Putaminal hyperintensity in proton density MRI was found in cases 5, 14, and 20 to 28. Cases 10,12,17,18, and 19 were not examined.

could not be assessed. Unilateral extrapyramidal signs were seen in two cases. Autonomic dysfunction was noted in all patients.

On T2 weighted imaging, decreased signal intensity of the putamen was greater at $1.5 \mathrm{~T}$ than $0.5 \mathrm{~T}$. On the other hand, slit-hyperintensity in the outer margin of the putamen was clear in both 1.5 and $0.5 \mathrm{~T}$ images. This slit-hyperintensity (figure; B,C,D) was found in 17 out of 28 cases of multiple system atrophy, 13 of whom showed extrapyramidal signs. The other four cases with slit-hyperintensity had no extrapyramidal signs, and one case with mild rigidity showed no hyperintensity. Unilateral slit-hyperintensity was seen in five cases. Among them, four cases showed contralateral rigidity, and one case bradykinesia (figure; C). On coronal sections, the slit-hyperintensity surrounded the hypointense putamen and reached to the outer margin of the caudate nucleus. One of 11 cases without slit-hyperintensity (figure A) presented no extrapyramidal signs. No cases of Parkinson's disease showed slithyperintensity in the lateral margin of the putamen.

On T1 weighted MRI, the density of the outer margin of the putamen was decreased in six out of 14 cases with extrapyramidal signs (figure; E). Hyperintensity was also seen in proton density MRI in eight out of nine cases with, and in two out of 13 without extrapyramidal signs (figure; $\mathbf{F}$ ).

The degree of slit-hyperintensity significantly paralleled the severity of bradykinesia $(r=0.8556, \mathrm{p}<0.001)$ and rigidity $(r=$ $0.8775, \mathrm{p}<0.001)$ when analysed by the least squares method. ${ }^{10}$ The degree of atrophy in the pontocerebellar system 


\begin{tabular}{|c|c|c|c|c|c|c|c|c|c|c|c|c|c|}
\hline \multirow{2}{*}{$\begin{array}{l}\text { Patient } \\
\text { No }\end{array}$} & \multirow{2}{*}{ Sex } & \multirow{2}{*}{ Age } & \multirow{2}{*}{$\begin{array}{l}\text { Initial } \\
\text { symptom }\end{array}$} & \multicolumn{2}{|c|}{$\begin{array}{l}\text { Duration of } \\
\text { symptoms }(y)\end{array}$} & \multicolumn{3}{|c|}{ Symptoms and signs } & \multirow{2}{*}{$\begin{array}{l}\text { Truncal } \\
\text { ataxia }\end{array}$} & \multirow[b]{2}{*}{ Incoordination } & \multicolumn{2}{|c|}{ Linear hyperintensity } & \multirow{2}{*}{$\begin{array}{l}\text { Pontocerebellar } \\
\text { atrophy }\end{array}$} \\
\hline & & & & $B$ & $A$ & $\mathrm{OH}$ & $B$ & Rigidity & & & Right & Left & \\
\hline 1 & $\mathbf{M}$ & 50 & A & 0 & 1 & + & - & - & + & + & - & - & + \\
\hline 2 & $\mathbf{F}$ & 48 & A & 0 & 1 & + & - & - & + & + & - & - & + \\
\hline 3 & $\mathbf{M}$ & 59 & A & 0 & 2 & + & - & - & ++ & + & - & + & + \\
\hline 4 & M & 69 & OH & 0 & 2 & ++ & - & - & ++ & + & - & - & ++ \\
\hline 5 & $\mathbf{F}$ & 62 & $\mathrm{OH}$ & 0 & 2 & + & - & - & +++ & + & - & - & + \\
\hline 6 & $\mathbf{F}$ & 52 & A & 0 & 3 & + & + & - & + & + & + & - & ++ \\
\hline 7 & $\mathrm{~F}$ & 55 & OH & 0 & 3 & +++ & - & - & +++ & ++ & - & - & ++ \\
\hline 8 & $\mathbf{M}$ & 62 & A & 0 & 3 & & - & - & +++ & + & - & - & +++ \\
\hline 9 & M & 53 & A & 0 & 4 & ++ & - & - & ++ & ++ & - & - & +++ \\
\hline 10 & $\mathbf{F}$ & 59 & A & 0 & 4 & ++ & - & - & +++ & +++ & + & + & +++ \\
\hline 11 & $\mathbf{M}$ & 59 & A & 0 & 5 & +++ & - & - & +++ & +++ & - & - & +++ \\
\hline 12 & $\mathbf{F}$ & 51 & A & 0 & 6 & ++ & - & - & +++ & +++ & + & + & +++ \\
\hline 13 & $M$ & 54 & $\mathrm{OH}$ & 0 & 6 & +++ & - & - & +++ & +++ & - & - & +++ \\
\hline 14 & $\mathbf{F}$ & 52 & A & 0 & 7 & ++ & - & - & +++ & +++ & - & - & +++ \\
\hline 15 & $\mathbf{F}$ & 61 & A & 0 & 7 & + & - & - & +++ & ++ & - & + & ++++ \\
\hline 16 & $\mathbf{M}$ & 57 & A & 1 & 4 & ++ & ++ & + & +++ & +++ & - & - & +++ \\
\hline 17 & F & 66 & A & 1 & 4 & + & + & + & +++ & ++ & + & + & ++ \\
\hline 18 & $\mathbf{M}$ & 63 & $\mathrm{OH}$ & 2 & 3 & ++ & +++ & ++ & +++ & ++ & ++ & ++ & +++ \\
\hline 19 & $\mathrm{~F}$ & 58 & B & 3 & 2 & ++ & ++++ & ++++ & +++ & + & ++ & + & \\
\hline 20 & $\mathbf{F}$ & 65 & $\mathbf{B}$ & 3 & 1 & + & ++ & ++ & + & + & ++ & ++ & + \\
\hline 21 & $\mathbf{M}$ & 54 & B & 4 & $?$ & + & +++ & ++ & - & - & + & + & ++ \\
\hline 22 & $M$ & 54 & $\overline{\mathbf{B}}$ & 4 & $?$ & ++ & ++ & $\mathbf{L}+, \mathbf{R}-$ & +++ & ++ & + & - & +++ \\
\hline 23 & $\mathbf{F}$ & 56 & B & 4 & 2 & + & ++ & $\bar{L}+++, \mathbf{R}-$ & ++ & + & ++ & - & \\
\hline 24 & $\mathrm{~F}$ & 59 & B & 4 & $?$ & ++ & ++ & $\mathrm{L}++, \mathbf{R}+$ & + & + & +++ & ++ & + \\
\hline 25 & $\mathbf{M}$ & 61 & B & 4 & 2 & ++ & ++++ & ++ & +++ & +++ & ++ & ++ & +++ \\
\hline 26 & M & 66 & B & 4 & ? & ++ & +++ & +++ & + & + & ++ & ++ & \\
\hline 27 & $\mathrm{~F}$ & 67 & B & 5 & $?$ & + & ++++ & ++++ & - & - & ++ & +++ & ++ \\
\hline 28 & $\mathbf{F}$ & 59 & $\mathbf{B}$ & 6 & $?$ & + & ++++ & ++++ & - & - & +++ & +++ & ++ \\
\hline
\end{tabular}

$\mathrm{A}=$ ataxia; $\mathrm{B}=$ bradykinesia; $\mathrm{OH}=$ orthostatic hypotension; $-=$ none; $+=$ mild; $++=$ moderate; $+++=$ severe; $++++=$ extremely severe.

correlated significantly with the severity of truncal ataxia $(r=0.8400, \mathrm{p}<0.001)$, but not with that of bradykinesia or rigidity.

\section{Discussion}

The main pathological features of multiple system atrophy are atrophy of the inferior olive, pons, and cerebellum as well as degeneration in the substantia nigra, putamen, and the intermediolateral cell columns and Onuf's nucleus of the spinal cord. ${ }^{11}$ Although such involvement can be subclinical in life,,$^{12}$ pathological features of multiple system atrophy are ultimately found in multiple systems. In our study, diagnostic criteria for multiple system atrophy comprised symptoms or signs in at least two of the cerebellar, extrapyramidal, and autonomic areas. When cerebellar symptoms were not evident clinically, atrophy of the pontocerebellar system in magnetic resonance imaging was accepted as evidence of cerebellar involvement.

The pathological changes in the striatum of striatonigral degeneration type multiple system atrophy comprise atrophy, neuronal loss, gliosis, and deposition of lipofuscin. ${ }^{2}$ This deposition of lipofuscin granules containing iron results in decreased signal intensity on T2 weighted MRI. ${ }^{5-7}$ The atrophy of the putamen is also usually severe, and this structure is often reduced in size to $30 \%$ of that in normal subjects. ${ }^{3}$ In this study, slit-hyperintensity in the lateral margin of the putamen was often seen in cases with striatonigral degeneration type multiple system atrophy with extrapyramidal signs, and the degree of this hyperintensity paralleled the severity of rigidity. Those patients with hemirigidity showed contralateral slit-hyperintensity. By contrast, no such abnormal hyperintensity was found in the patients with clinically diagnosed Parkinson's disease. These results suggest that this abnormal hyperintensity relates directly to extrapyramidal dysfunction in patients with striatonigral degeneration type multiple system atrophy. The three patients who showed mild slit-hyperintensity but no extrapyramidal signs may have subclinical striatonigral degeneration, and will show these signs at some future date.

The hyperintense lines we have found follow the lateral margin of the putamen, and often reach the head of the caudate nucleus. Hyperintensity on T2 weighted images is generally considered to be due to demyelination, gliosis, or increased extracellular fluid. The increased signal intensity on T2 weighted images in the lateral margin of the putamen is considered to be due to an increase in extracellular fluid caused by severe atrophy of the putamen and enlargement of the space between the putamen and external capsule. The most pronounced involvement of the putamen in striatonigral degeneration is in its posterior dorsolateral part, where severe gliosis may result in increased hyperintensity on proton density images.

In the present cases of multiple system atrophy with extrapyramidal signs, slit-hyperintensity in the outer margin of the putamen is a common sign, which suggests that this change relates to damage to the basal ganglia. This radiological feature is useful for the differential diagnosis between striatonigral degeneration type multiple system atrophy and Parkinson's disease, and for estimating the severity of the striatal lesion in striatonigral degeneration type multiple system atrophy. 
1 Graham JG, Oppenheimer DR. Orthostatic hypotension and nicotine sensitivity in a case of multiple system atrophy. $\mathcal{F}$ Neurol Neurosurg Psychiatry 1969;32:28-34.

2 Adams RD, van Bogaert L, Vandereecken $H$. Dégénérescences nigro-striées et cérébello-nigro strée. Psychiatr Neurol 1961;142:219-259.

3 Fearnley JM, Lees AJ. Striatonigral degeneration. A clinicopathological study. Brain 1990;113:1823-42.

4 Kume A, Takahashi A, Hashizume Y, et al. A histochemical and comparative study on Purkinje cell loss and olivary nucleus cell loss in multiple system atrophy. $\mathcal{F}$ vary nucleus cell loss in mul
Neurol Sci 1991;101:178-86.

5 Drayer BP, Olanow W, Burger P, et al. Parkinson plus rayer BP, Olanow W, Burger P, et al. Parkinson plus
syndrome. Diagnosis using high field MR imaging of brain iron. Radiology 1986;159:493-8.

6 Pastakia B, Polinsky R, Di Chiro G, et al. Multiple system atrophy (Shy-Drager syndrome). MR imaging. Radiology 1986;159:499-502.

7 O'Brien C, Sung JH, McGeachie RE, Lee MC.
Striatonigral degeneration. Clinical, MRI, and pathologic correlation. Neurology 1990;40:710-1

8 Savoiardo M, Strada L, Girotti F, et al. MR imaging in progressive supranuclear palsy and Shy-Drager syndrome. $\mathcal{F}$ Comput Assist Tomogr 1989;13:555-60.

9 Savoiardo M, Strada L, Girotti F, et al. Olivopontocerebellar atrophy. MR diagnosis and relationship to multisystem atrophy. Radiology 1990;174: 693-6.

10 Ichihara K. Simple linear regression. In: statistics for bioscience. Tokoyo: Nanko-do, 1990;206-11.

11 Oppenheimer DR. Diseases of the basal ganglia, cerebellum and motor neurons. In: Adams JH, Corsellis JAN, Duchen LW, eds. Greenfield's neuropathology, 4th ed. Duchen LW, eds. Greenfield's neuropath.

12 Bruyn GW, Bruyn RPM. Striatonigral degeneration Entity or non-entity? In: Vinken PJ, Bruyn GW, Klawans HL, eds. Handbook of clinical neurology. Vol 16(60). Hereditary neuropathies and spinocerebellar atrophies. Amsterdam: Elsevier, 1991:537-49. 